\title{
The isopropyl ester of methionine hydroxy-analogue is absorbed through the rumen wall in the cow
}

\author{
B. Graulet ${ }^{1}$, C. Richard $^{1}$ and J.C. Robert ${ }^{2,3}$ \\ ${ }^{1}$ CERN, ADISSEO France SAS \\ 03100 Commentry, France \\ ${ }^{2}$ Research and Development Department, ADISSEO France SAS \\ 92160 Antony, France
}

\section{ABSTRACT}

A new form of methionine, the isopropyl ester of methionine hydroxy analog (HMBi), has been proven to be available for milk protein synthesis in dairy cows. Studies have also shown that HMBi can be degraded by rumen micro-organisms. By two different experimental approaches (emptied and washed rumen; catheterization of the ruminal vein), we have demonstrated that HMBi ability to be rapidly absorbed by the rumen wall can explain its partial protection from microbial degradations. The high efficiency of HMBi to pass through biological membranes seems to be due to the molecule's isopropyl ester radical.

KEY WORDS: rumen-protected methionine, ruminal absorption, methionine hydroxylanalog ester, bioavailability

\section{INTRODUCTION}

Methionine (Met) requirements are not covered in the lactating dairy cows when rumen-undegradable proteins are provided by legume, soya products or animal meal (NRC, 2001). Adequate methionine supply is especially important for dairy cows, both for milk protein synthesis and for improvement of nitrogen utilization. Thus, manufacturers have developed protection methods against rumen bacterial degradations such as coating or chemical modification of the Met molecule. HMBi has been proven to be a good form of metabolizable Met for the dairy cows (Robert et al., 2001a; Schwab et al., 2001; Sylvester et al., 2003). However, a recent in vitro test also showed that the isopropyl ester of methionine hydroxy-analogue (HMBi) can be hydrolysed into HMB and isopropyl alcohol by rumen micro-organisms (Robert et al., 2002). This result is consistent with those of Patterson and Kung (1988) on certain other Met chemical derivatives. Thus, we wanted to understand this apparent

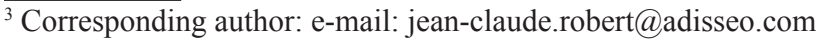


contradiction: how HMBi could be both available for milk protein synthesis by the dairy cows and a good substrate for rumen microflora at the same time. For this purpose, we develop two different exploratory experimental approaches: the emptied and washed rumen to characterize the HMBi ability to pass through the rumen wall and the ruminal vein catheterization to demonstrate that HMBi is absorbed efficiently by the rumen wall even in the presence of the micro-organisms.

\section{MATERIAL AND METHODS}

\section{Study 1. Ability of HMBi to pass through the rumen wall (emptied and washed rumen test)}

We tested the ability of HMBi to pass through the rumen wall, by comparison with DL-Met or HMB. The emptied and washed rumen technique was applied to two dry non-gestating Jersey cows (mean body weight: $472 \pm 6 \mathrm{~kg}$; age: 7 years) fitted with a rumen canula, and a jugular catheter. Each of the three items was tested on the two cows except HMBi for which one repetition is missing. A 4-L buffer solution containing $50 \mathrm{~g}$ Met equivalent of item (HMBi, HMB or DL-Met) was incubated for $3 \mathrm{~h}$ into the emptied and washed rumen closed with a smooth rubber plug in the reticulo-omasal orifice (Kristensen et al., 2000). Blood samples were taken (16 samplings per cow and per item) from the day before the experiment until 2 days after incubation started. HMB and Met were assayed by high performance liquid chromatography after deproteinization of the blood plasma. Met concentrations were used to calculate Areas Under Curves (AUC) and Met bioavailability of the items using the relationship between increasing spot digestible Met (MetDi) supplies and plasma Met responses in term of AUC (Robert et al., 2001b). Due to the poor number of repetitions and incomplete design, no statistical analysis was realized.

\section{Study 2. HMBi absorption through the rumen wall (functional rumen test)}

We tested HMBi absorption by the rumen wall in the presence of micro-organisms by catheterization of both the ruminal and jugular veins of two Holstein cows (mean BW: $678 \pm 20 \mathrm{~kg}$; age: 5 years). Plasma HMBi, HMB and Met concentrations were determined (16 samplings per cow) after rumen HMBi supplementation (50 g met equivalent). Met concentrations were used to calculate AUC and Met bioavailability of the items according to a standard curve (Robert et al., 2001b).

\section{RESULTS}

Study 1. Ability of HMBi to pass through the rumen wall (emptied and washed rumen test)

Under the experimental conditions of emptied and washed rumen, plasma HMB concentrations at the jugular level peaked quickly (in the 30 first min) after the start 
of HMBi incubation, going from 0 to $22.15 \mathrm{mg} / 100 \mathrm{~g}$ of plasma. Met concentrations also increased rapidly from 0.33 to $5.11 \mathrm{mg} / 100 \mathrm{~g}$ at peak which occurred during the incubation period. Thereafter, Met concentrations decreased slowly to restore the basal level $48 \mathrm{~h}$ after incubation started.

When HMB was incubated, plasma HMB concentration at peak was 15-fold lower than the corresponding value observed after HMBi supplementation, and Met concentration was very little affected.

Finally, when DL-Met was incubated in the rumen, plasma Met concentration varied poorly from 0.38 to $1.79 \mathrm{mg} / 100 \mathrm{~g}$ at peak.

AUC were calculated; MetDi and Met availability of the products were deduced using a calibration curve (Robert et al., 2001b).

Table 1. Evaluation of HMBi, HMB and DL-Met bioavailability

\begin{tabular}{lccccc}
\hline Item & Cow & AUC & $\begin{array}{c}\text { MetDi } \\
\mathrm{g}\end{array}$ & $\begin{array}{c}\text { Met availability } \\
\%\end{array}$ & $\begin{array}{c}\text { Met availability } \\
\text { (mean } \pm \text { se), \% }\end{array}$ \\
\hline HMBi & A & 74.5 & $\begin{array}{c}45.4 \\
\text { nd }\end{array}$ & $\begin{array}{c}90.5 \\
\text { nd }\end{array}$ & \\
& B & nd & & 13.9 & $16.5 \pm 2.6$ \\
HMB & A & 5.41 & 7.0 & 19.1 & \\
& B & 7.95 & 9.5 & 16.1 & $19.1 \pm 3.0$ \\
& A & 6.61 & 8.0 & 22.0 & \\
\hline
\end{tabular}

nd - not determined, se - standard error of mean

${ }^{1}$ - Met availability $=($ MetDi/50 g Met equivalent supplied $) \times 100$

\section{Study 2. HMBi absorption through the rumen wall (functional rumen test)}

Plasma HMBi, HMB and Met concentrations determined in kinetics after HMBi supplementation (50 g met equivalent) showed that HMBi was rarely and extremely transiently detectable in the ruminal vein and completely absent from the jugular vein. By contrast, peak values of plasma HMB concentrations were almost concomitant in both veins and appeared very quickly (at 11 and 32 min after supplementation for the two cows, respectively), but they were higher in the ruminal than in the jugular vein $(8.53 \pm 0.19$ vs $4.62 \pm 0.19 \mathrm{mg} / 100 \mathrm{~g}$, respectively) demonstrating a ruminal absorption of HMBi even in presence of micro-organisms in the rumen.

\section{DISCUSSION}

HMBi appears in the ruminal vein mostly in the form of HMB. The hydrolysis of $\mathrm{HMBi}$ into $\mathrm{HMB}$ was probably post-absorptive because HMB absorption by the rumen wall is very limited ; thus, it is highly unlikely that the HMB 
appearing in the ruminal vein could come from HMBi hydrolysed to HMB prior to absorption. Moreover, plasma HMB peak times after HMB supplementation in a microbiologically functional rumen indicated a duodenal absorption of HMB (14:30 $\mathrm{h}$ after supplementation) whereas they were consistent with a ruminal absorption for HMBi (1:11 h after supplementation) (Robert et al., 2001c). The differences between sites of absorption of HMB and HMBi could be related to their physico-chemical properties, particularly their hydrophile-lipophile balance, with HMBi having a greater capacity to penetrate in biological membranes.

In spite of the fact that these studies were mainly exploratory and need complementary results and observations, for the first time, we demonstrated that $\mathrm{HMBi}$ is able to be both quickly and effectively absorbed by the rumen wall and transferred to the blood plasma compartment.

This quick rumen escape, via absorption by the rumen wall, appears to be the primary means of protection for HMBi against microbial degradation. This explains how, to a certain extent, HMBi can be both bio-available and rumen degradable.

\section{REFERENCES}

Kristensen N.B., Gäbel G., Pierzynowski S.G., Danfær A., 2000. Portal recovery of short-chain fatty acids infused into the temporarily-isolated and washed reticulo-rumen of sheep. Brit. J. Nutr. $84,477-482$

National Research Council, 2001. Nutrient Requirements of Dairy Cattle. $7^{\text {th }}$ revised Edition. Natl. Acad. Press, Washington, DC

Patterson J.A., Kung L. Jr., 1988. Metabolism of DL-methionine and methionine analogs by rumen micro-organisms. J. Dairy Sci. 71, 3292-3301

Robert J.C., Richard C., Bouza B., 2001a. Influence of monomer or dimer forms of isopropyl ester of HMB, on the supply of metabolisable methionine to the blood of ruminants. J. Dairy Sci. 84, Suppl. 1, 281

Robert J.C., Etave G., D'Alfonso T., Bouza B., 2001b. A blood kinetics methodology to measure bioavailability of rumen protected methionine sources for ruminants. J. Dairy Sci. 84, Suppl. 1, 281

Robert J.C., Richard C., D'Alfonso T., Ballet N., Depres E., 2001c. Investigation of the site of absorption and metabolism of a novel source of metabolisable methionine: 2-hydroxy-4 (methylthio) butanoic acid isopropyl ester. J. Dairy Sci. 84, Suppl. 1, 35

Robert J.C., Ballet N., Richard C., Bouza B., 2002. Ruminal metabolism of 2-hydroxy-4 (methylthio) butanoic acid isopropyl ester (HMBi). J. Dairy Sci. 85, Suppl. 1, 240

Schwab C.G., Whitehouse N.L., McLaughlin A.M., Kadariya R.K., St-Pierre N.R., Sloan B.K., Gill R.M., Robert J.C., 2001. Use of milk protein concentrations to estimate the "methionine bioavailability" of two forms of 2-hydroxy-4-methylthio butanoic acid (HMB) for lactating cows. J. Dairy Sci. 84, Suppl. 1, 35

Sylvester J.T., St-Pierre N.R., Sloan B.K., Beckman J.L., Noftsger S.M., 2003. Effect of HMB and HMBi on milk production, composition, and $\mathrm{N}$ efficiency of Holstein Cows in early and midlactation. J. Dairy Sci. 86, Suppl. 1, 60 